http:/ / dx.doi.org/10.21707/gs.v11.n03a06

\title{
Produção E USO doS RECURSOS MELÍfEROS POR MELIPONICULTORES DA região de Cícero Dantas, BA
}

\author{
Lidiane Nunes Lima ${ }^{1 *}$, Eliane Maria de Souza Nogueira ${ }^{2}$
}

\author{
${ }^{1}$ Mestre em Ecologia Humana e Gestão Socioambiental, Universidade do Estado da Babia. \\ ${ }^{2}$ Docente do Programa de Pós-Graduação stricto sensu em Ecologia Humana e Gestão Socioambiental, Universidade do Estado da Babia. \\ *Autor para correpondência: lidiane.linu@gmail.com
}

Recebido em 08 de junho de 2016. Aceito em 23 de abril de 2017. Publicado em 29 de julho de 2017.

\begin{abstract}
Resumo - As abelhas da tribo Meliponini são conhecidas como abelhas sem ferrão e estão representadas por aproximadamente 250 espécies no Brasil. Muitas espécies são criadas para a produção de mel. Dada a importância destas abelhas para o semiárido brasileiro, o presente estudo tem como objetivo descrever os aspectos da produção do mel pelos meliponicultores da região de Cícero Dantas-BA, além de identificar os valores culturais da comunidade associadas a esta prática. Para este fim, foram realizadas 21 entrevistas semiestruturadas entre os meses de março e dezembro de 2014, com meliponicultores da região. Constatou-se que os mesmos comercializam e consomem preferencialmente o mel das abelhas nativas que criam, utilizando também o pólen e a resina. A retirada do mel está condicionada a quantidade produzida, à procura para compra e a sua utilização. Além de ser comercializado, o mel é também utilizado para fins terapêuticos, principalmente no tratamento de gripes e resfriados. A atividade da criação de abelha, bem como o uso dos produtos derivados da atividade dos meliponíneos está estritamente relacionada com os valores culturais da região, que são perpassados de geração a geração. A prática da meliponicultura além de ser uma alternativa econômica sustentável, também pode ser tida como uma opção para a conservação de espécies da flora e fauna nativas.
\end{abstract}

Palavras chave: Meliponicultura; Nordeste; Abelhas Nativas; Valores Culturais.

Production and use of honey resources by stingless beekeepers in the region of Cicero Dantas, Bahia

Aвstract - Bees of the Meliponini tribe, known as stingless bees, are represented by approximately 250 species in Brazil. Many species are grown for the production of honey. Given the importance of these bees to the Brazilian Semi-arid region, the present study aims to describe aspects of the honey production by beekeepers from the area of Cícero Dantas, Bahia, besides identifying community cultural values associated with this practice. For this purpouse there were 21 semistructured interviews conducted between March and December 2014, with beekeepers in the region. It was found that the beekeepers sell and use, preferably the native bees' honey grown by them, also using pollen and the resin created. The withdrawal of honey is related to how much it's produced, the demand for purchase and use. Besides comercialized, honey is also used for therapeutic purposes, especially in the treatment of colds and flus. The activity of growing bees and the use of products derived from this activity are closely related to the cultural values of the region, that are passed on generation to generation. The practice of beekeeping in addition to being aneconomical sustainable alternative, can also be taken as an option for the conservation of native species of flora and fauna.

Keywords: Meliponiculture; Northeast; Native bees; Cultural V alues.

Producción y uso de los recursos melíferos por meliponicultores de la región de Cícero DaNTAs, BA

REsumen - Las abejas de la tribu Meliponini son conocidas como abejas sin aguijón y están representadas por 
aproximadamente 250 especies en Brasil. Muchas especiesson criadas para la producción de miel. En función de su importancia para la región semiárida de Brasil, este estudio tiene como objetivo describir los aspectos de la producción de la miel por los meliponicultores de la región de Cicero Dantas, Bahia, además de identificar los valores culturales de la comunidad asociados a esta práctica. Para este fin, se llevaron a cabo 21 entrevistas semiestructuradas entre los meses de marzo y diciembre de 2014, con meliponicultores de la región. Se constató que los mismos comercializan y consumen preferencialmente la miel de las abejas nativas que crían, utilizando tambiénel polen y la resina. La extracción de la miel está condicionada a la cantidad producida, a la demanda de compray a su utilización. Además de ser comercializada, la miel es también utilizadacon fines terapéuticos, especialmente en el tratamiento de resfriados y gripe. La actividad de la producción de abejas, así como el uso de productos derivados de la actividad de los meliponicultores está estrechamente relacionada a los valores culturales de la región, que son transmitidos de generación en generación. La práctica de la meliponicultura, además de ser una alternativa económica sostenible, también puede ser tomada como una opción para la conservación de especies de flora y fauna nativas.

Palabras clave: Meliponicultura; Nordeste; Abejas Nativas; V alores culturales.

\section{INTRODUÇÃO}

As abelhas nativas são responsáveis pela polinização de grande parte dos ecossistemas brasileiros, incluindose cerca de 30\% das espécies da caatinga e do pantanal, chegando até $90 \%$ em algumas manchas de mata atlântica e algumas partes da Amazônia (Kerr 1997). Tal evento é relevante para a natureza, visto que contribui para a melhoria da produção de sementes e frutos (Kerr et al. 1996; Imperatriz-Fonseca et al. 2004). Esse papel ecológico das abelhas garante a propagação de muitas espécies nativas, evitando desse modo, a extinção de espécies de plantas importantes para os ecossistemas, ora tão degradados. Além da contribuição ecológica, as abelhas desempenham um papel muito importante em diversas culturas humanas, ultrapassando a importância econômica, agregando valores simbólicos, místicos e cosmológicos (Camargo e Posey 1990; Posey 1983).

As abelhas produzem mel, cera, própolis e resina. Esses produtos são comumente utilizados para alimentação, para tratamento de doenças e preparação de bebidas (Posey 1978; Posey 1982; Carvalho et al. 2014). No semiárido brasileiro, onde o extrativismo de mel de abelhas nativas é uma prática tradicional dos sertanejos (Lopes et al. 2005), a criação de abelhas nativas, conhecida como meliponicultura contribui diretamente para a conservação das abelhas e seus habitats (Kerr et al. 1996). No Brasil, ela é mantida principalmente por povos indígenas, comunidades tradicionais e camponeses (Alves et al. 2007) e, constitui-se numa alternativa econômica para comunidades agrícolas familiares, através da venda dos produtos como o mel e o pólen, além de aumentar à produtividade das lavouras próximas as regiões de criação (Venturieri et al. 2003).

Além da diversidade biológica, a meliponicultura associa-se a uma grande quantidade de práticas e saberes locais, que marcam a relação entre o âmbito biofísico e o sociocultural, tendo implicações na conservação, manejo e gestão da fauna nativa (Nates-Parra e Rosso-Londoño 2013), atendendo os preceitos do uso sustentável dos recursos naturais, uma vez que não necessita da remoção da cobertura vegetal (Venturieri 2008). Sendo assim, o presente trabalho tem como objetivo registrar os aspectos da produção de mel pelos meliponicultores da região de Cícero Dantas-BA, bem como descrever suas práticas de uso e seus valores culturais.

\section{Material e MÉtodos}




\section{Descrição da área}

O município de Cícero Dantas está localizado no nordeste da Bahia, distante $301.00 \mathrm{~km}$ de Salvador,

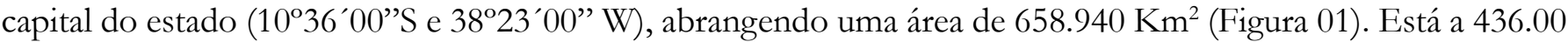
m de altitude em relação ao nível do mar, numa área de clima de subúmido a seco, típico do bioma caatinga brasileiro, com temperatura média anual de $23.1^{\circ} \mathrm{C}$, precipitação pluviométrica média no ano de $901,9 \mathrm{~mm}$ e período chuvoso de maio a julho (SEI-BA 2011). A população estimada é de 32.304 habitantes, dos quais 17.826 pessoas residem na área urbana e 14.578 na área rural (IBGE, 2010).

O tipo de vegetação predominante é a caatinga (SEI-BA 2011), caracterizada como florestas arbóreas ou arbustivas, com a presença principalmente de árvores e arbustos baixos, muitos dos quais apresentam espinhos, microfilia e algumas características xerofíticas (Prado 2003). As receitas municipais e renda da população provêm basicamente da agricultura, pecuária e avicultura. Na agricultura destaca-se a produção expressiva de feijão e milho. Os maiores rebanhos são os bovinos, suínos e ovinos. O município produz ainda mel de abelhas do tipo Apis e leite de vaca (SEI-BA 2011).

Figura 01 - Localização do município de Cícero Dantas-Bahia.
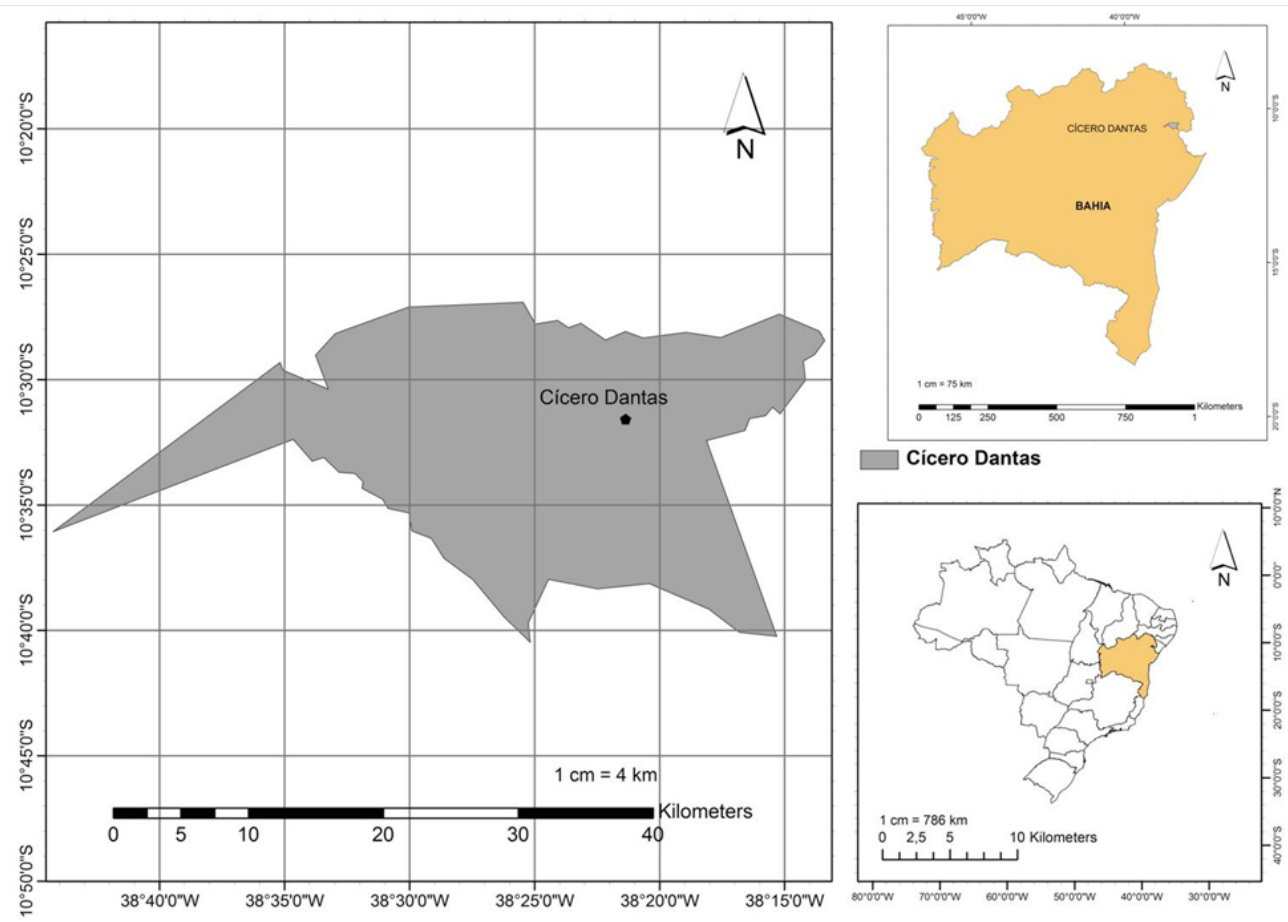

Coleta análise dos dados

A pesquisa de campo foi realizada entre os meses de março e dezembro de 2014. A escolha dos participantes da pesquisa foi realizada através da técnica de amostragem Snowball "bola de neve", onde um primeiro meliponicultor foi entrevistado, que passou a indicar outras pessoas da área estudada que praticavam a meliponicultura, repetindo-se o processo com os demais participantes até que as indicações se repetissem, conforme Bailey (1994). Para a coleta de dados foi utilizada entrevista semiestruturada, que possibilita uma maior flexibilidade as perguntas que foram aplicadas (Albuquerque et al. 2010), dentro da temática foi estabelecido como pontos norteadores: aspectos da produção do mel e identificação dos valores culturais da comunidade associadas a esta prática. Foram entrevistados 21 meliponicultores residentes em localidades rurais e imediações 
da área urbana da cidade, com idades que variaram entre 22 e 82 anos.

As entrevistas foram gravadas com o auxílio de uma câmera filmadora. Para isso, foi assinado o termo de concessão de imagem e som pelos informantes. Vídeos e transcrições das entrevistas estão depositados no Núcleo de Estudos em Povos e Comunidades Tradicionais e Ações Socioambientais (NECTAS), Universidade do Estado da Bahia. A pesquisa foi submetida e aprovada pelo comitê de ética da Universidade do Estado da Bahia-UNEB, parecer $n^{\circ} 541.644$, e o consentimento dos informantes para publicação dos resultados e quaisquer imagens que o acompanham foi obtido através do Termo de Consentimento Livre e Esclarecido (TCLE).

Para identificação das plantas visitadas e usadas pelas abelhas como local de nidificação na natureza, construção de cortiços, bem como fontes de néctar, pólen e resina, foi utilizado o método de turnê guiada (Albuquerque et al. 2010), que consiste na excursão numa área de vegetação com um dos representantes da comunidade para que o mesmo informe os nomes vernáculos de plantas e abelhas que ocorrem na região, bem como suas utilidades. As espécies de plantas apontadas foram fotografadas e seus nomes vernáculos anotados. Para a identificação das espécies seguiu-se a metodologia do tipo pista taxonômica (Martins 2011), que consiste na comparação dos nomes vernáculos citados com trabalhos da mesma região, bem como com catálogos de flora.

Foram coletados exemplares das espécies de abelhas citadas pelos meliponicultores, as mesmas passaram por preparação e montagem para exposição em caixa entomológica e identificadas por especialista da área e encontram-se depositados no laboratório de ecologia animal da UNEB - Universidade do Estado da Bahia.

Os dados foram analisados seguindo a abordagem qualitativa proposta por Amorozo e Viertler (2010), complementando com a análise de conteúdo das falas de acordo com Bardin (2011). O material selecionado para representar este artigo foi organizado e descrito na forma de texto, seguindo a abordagem êmica e ética, onde o contexto êmico é discutido de acordo com a visão dos meliponicultores, e ético segundo a interpretação da cultura estudada pelos pesquisadores.

\section{Resultados E Discussão}

Aspectos da produção do mel, pólen e resina de abelhas sem ferrão

Na região rural de Cícero Dantas foram identificadas sete espécies de abelhas nativas sem ferrão manejadas pelos meliponicultores locais, dentre elas a Mandaçaia (Melipona mandacaia), criada por 21 meliponicultores, a Moça branca (Frieseomelitta doederleini) 5 meliponicultores, Mosquitinho (Plebeia sp.) 7 meliponicultores, Jataí (Tetragonisca angustula) e Manduri (Melipona asilvar) 2 meliponicultores, Caruara (Trigona sp.) e Cupinheira (Partamona sp.), apenas 1 meliponicultor. A maior ocorrência de criação da espécie mandaçaia pode ser justificada pelo ótimo sabor e maior produtividade de mel em relação às demais espécies. A quantidade de enxames por meliponicultor variou entre 46 e apenas três, porém a grande maioria possuía entre seis e 20 enxames de abelhas nativas.

Nos últimos anos muitas espécies de abelhas sem ferrão tem sido criadas com diversas finalidades, no entanto, a principal ainda é a criação para produção de mel, como é o caso das espécies Melipona scutellaris, $M$. fasciculata, M. quadrifasciata, M. subnitida, Tetragonisca angustula e Scaptotrigona depilis, dentre outras (Kerr et al. 2006; Venturieri et al. 2003; Yamamoto et al. 2007; Cortopassi-Laurino 2005).

A forma de criação é distinta entre os meliponicultores, no entanto, verifica-se que há aqueles que a mantém 
de forma tradicional optando pela manutenção das abelhas em "cortiços", nomenclatura utilizada para designar troncos de árvores com diâmetro e tamanho especifico para o desenvolvimento dos ninhos das abelhas de acordo com as espécies. Outros optam por caixas mais tradicionais, que em sua maioria apresenta apenas um compartimento, assemelhando-se às estruturas encontradas na natureza. Por fim alguns preferem a utilização de caixas racionais, que apresentam várias subdivisões internas com a finalidade de melhorar o desenvolvimento dos enxames, aumentar a produção de mel e facilitar o manejo (Figura 02).

Figura 02 - Locais de criação das abelhas nativas. A - Caixas racionais modelo IMPA, utilizadas pelos meliponicultores para a criação de abelhas nativas; B - Caixas rústicas utilizadas na criação de abelhas nativas; C- Cortiços utilizados pelos meliponicultores para a criação das abelhas.

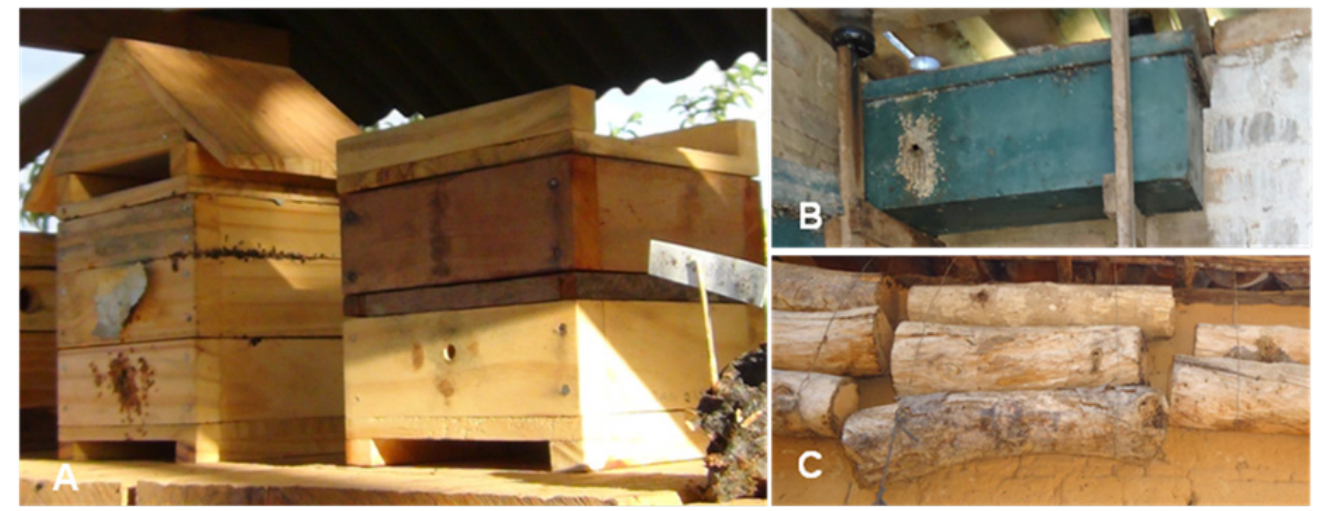

Segundo os informantes o mel é o principal produto de interesse dos meliponicultores de Cícero DantasBA. Em média é relatada a retirada de $300 \mathrm{ml}$ a um litro de mel por enxame. Essa quantidade varia de acordo com a espécie criada e disponibilidade de flores melíferas nas áreas próximas aos enxames. Em várias regiões do Brasil a produção média anual de colônias bem estabelecidas é de quase um ou dois litros por ninho, em colmeias padrão. Para espécies como Melipona subnitida, registrou-se uma produção de cerca de 6,0 litros/ano, nos anos 80 em algumas colônias, durante a estação das chuvas nas regiões de caatinga do Brasil (CortopassiLaurino e Macêdo 1998).

A retirada é feita pela maioria dos criadores em períodos irregulares de tempo, que a condiciona à florada local. Dois meliponicultores confirmaram a retirada a cada seis meses e outros dois, apenas uma vez por ano, os demais retiram o mel de forma irregular, conforme fala de um entrevistado:

[...] so tiro nessa base de seis em seis meses [...] em ano em ano, quando o tempo ta ruim, a seca né, mas o tempo tando bom é de seis em seis meses, que tem a fulo né, a árvore do pau [...].

Nessa narrativa fica evidente que a quantidade de mel retirado pode variar de acordo com as condições climáticas, que condicionam à produção da florada das plantas da região. Quando o ano tem elevados índices pluviométricos, em detrimentos aos períodos de baixa pluviosidade, marcadamente pela seca, favorece a produção de flores melíferas na região. Os altos índices pluviométricos em certos meses do ano, principalmente entre novembro e janeiro, caracteriza o que os produtores de mel consideram como "trevoadas". O evento possibilita a florada das plantas melíferas da região favorecendo as abelhas na coleta de néctar e pólen, levando, consequentemente, a uma elevação na produção de mel. Estudos realizados na área por Lopes e Nogueira (2012) identificam comportamento semelhante, verificando que a melhor época para coleta do mel ocorreu logo após o período identificado pelas “trevoadas", citadas pelos meliponicultores da região. Esses resultados reforçam os argumentos de Villanueva et al. (2005), quando afirmam que a produção do mel de abelhas nativas 
depende diretamente da qualidade do ambiente, da concorrência por recursos florais com outras espécies, bem como do manejo das abelhas.

Segundo os meliponicultores, o mel muitas vezes não é retirado dos enxames devido à preocupação com fornecimento de alimento para as abelhas, uma vez que, ao retirar o mel dos enxames, as abelhas podem morrer por falta de suplemento energético. Um dos criadores informa que não retira o "saborá", termo utilizado pelos meliponicultores para nomear o pólen, pois o mesmo é uma das principais fontes alimentares das abelhas. Entre as abelhas do gênero Melipona, é importante garantir que a alimentação apresente um valor nutricional adequado ao seu metabolismo, pois a determinação das castas é influenciada pela qualidade do alimento que e despejado nas células de cria (Kerr et al. 1996).

Os meliponicultores vendem o mel de acordo com a quantidade que é retirada em cada coleta. Se a produção é muito pequena o mel não é vendido e fica para uso da família, amigos e parentes. Uma vez atingindo uma produção considerada grande pelos meliponicultores, o mel é comercializado e seu preço varia conforme a época e tipo de abelha. Há meliponicultores que vendem o litro do mel a $\mathrm{R} \$ 50,00$ (valor em 2015), outros, porém a mesma quantidade chega a ser vendida por $\mathrm{R} \$ 100,00$. O preço varia conforme a oferta/demanda, espécies que produzem mel em pequena quantidade ou são utilizados no tratamento de doenças, como a Jataí (Tetragonisca angustula) apresentam um maior valor de venda. Este fato não é restrito ao sertão baiano. Em outras regiões observa-se comportamento semelhante, seja no Brasil (Venturieri et al. 2003), ou fora do país, a exemplo do México (Blásquez et al. 2009).

A aplicação da renda obtida com a comercialização tem diversas finalidades: há quem a aplique na aquisição direta de bens, como uma vaca ou outro bovino, embora a maioria investida na compra de comida e produtos para a manutenção das residências. Apenas um relatou a compra de madeira para a construção de novas caixas para as abelhas e compra de água em épocas de seca da região.

No Brasil, especialmente em 2001 foi realizado um levantamento cujos resultados demonstraram que a meliponicultura está crescendo rapidamente (Rosso et al. 2001), aparecendo como uma atividade rentável nos últimos anos, onde pequenos frascos de mel de determinadas espécies de abelhas podem ser encontrados em mercados locais por preços elevados, superior ao praticado para o mel de abelhas africanizadas, como Apis, atingindo o valor de 40 dólares/litro (Cortopassi-Laurino et al. 2006). No entanto, para muitos que praticam a atividade, ela é considerada uma atividade econômica secundária.

No tocante a forma de comercialização verificou-se que é realizada diretamente aos interessados, a quantidade vendida está condicionada ao preço. A procura normalmente é feita diretamente ao criador ou através de encomenda prévia, como explica um dos meliponicultores:

A gente também não vende diretamente, só vende de pouco em pouco. Porque é muito caro e o pessoal não pode comprar, porque é muito caro [...].

Conforme Alves et al. (2007), pequenos produtores nordestinos que residem em comunidades rurais normalmente comercializam seu mel ao retirá-lo do cortiço. $\mathrm{O}$ mesmo pode ser visto na região estudada, a venda normalmente é feita uma vez por ano, o mel é entre diretamente ao interessado, o que não acontece com a cera e a resina, que normalmente é utilizada para fins pessoais. Em outras regiões do mundo a venda do mel, da cera e da resina contida nos ninhos das abelhas sem ferrão, é tida como uma alternativa financeira, outra opção é o arrendamento de colônias para o serviço de polinização (Cortopassi-Laurino et al. 2006). 
Uso do mel, pólen e resina pelos meliponicultores

Um aspecto muito importante na produção do mel no semiárido, também importante para o sertanejo, é o seu uso para tratamento de doenças, como enfermidades na boca, conhecidas como "sapinho" ou aftas bucais.

[...] pra doença, o mel, ele é cicatrizante, ao mesmo tempo é antibiótico, pra criança que tem, antigamente saia né, que o povo chamava de sapinho na boca de criança, tem quem já pegou e sarou rapidinho.

$\mathrm{Na}$ literatura especializa encontram-se dados que confirmam as indicações terapêuticas dos méis como antibióticos e fungicidas, conforme relato do meliponicultor. Torres et al. (2004) evidenciaram a ação do mel de Tetragonisca angustula, oriundo da Colômbia, através de testes com bactérias Gram-positivas (Bacillus brevis, Bacillus megaterium, Bacillus subtilis e Micrococcus luteus) e Gram-negativas (Escherichia coli e Pseudomonas syringae). Borsato et al. (2013) estudaram a atividade antibacteriana e antifúngica de méis in natura do Paraná, elaborados por abelhas nativas sem ferrão, a atividade antimicrobiana foi testada em Escherichia coli, Staphylococcus aureus e Candida albicans. Mercês et al. (2013), utilizando-se de teste de difusão em poço, estudaram a atividade antimicrobiana de méis produzidos por Melipona asilvai, M. quadrifasciata anthidioides, Friseomelita doederleinei, Tetragonisca angustula e Plebeia sp., demonstrando que todos os méis estudados tem ação antibacteriana frente a Staphylococcus aureus.

Outros autores ainda citam outras indicações, como Blásquez et al. (2009) citam o uso do mel no tratamento de tosses, bronquite, asma, além de doenças relacionadas a garganta, olhos e estômago, ou tônico geral. Badie (2009) descreve a utilização do mel da abelha Friesella schrottky (pyeguari mirì), pelos Mbye-Guraní na prevenção de aftas em recém-nascidos. Costa-Neto (1998) registra a utilização do mel de diversas espécies de abelhas e vespas pelos Pankararé no tratamento de diabetes, asma, bronquites, tuberculoses, verminoses, impotência dentre outras patologias. Ainda no nordeste há relatos da utilização para fins medicinais, mais precisamente no tratamento de "problemas de vista", onde ocorre a utilização do mel da abelha Cupira (Partamona sp) (Lopes et al. 2005).

Além do mel, o pólen também é descrito pelos meliponicultores locais como empregado na alimentação, podendo ser utilizado como alternativa no tratamento de gripes. Souza et al. (2004) relata que tanto o pólen, quanto o mel tem despertado a atenção do homem pela riqueza dos seus constituintes nutricionais, pois suas propriedades alimentícias tem grande valor na dieta humana, sendo o mel uma grande fonte de energia.

A utilização da cera das abelhas também foi relada na vedação de recipientes utilizados para armazenar grãos, mais conhecidos como "cilos". A utilização de cera para a conservação de produtos agrícolas foi citada por Sampaio et al. (2009) em um estudo com os Pankararé do Nordeste. Já entre os moradores do município de Tacotalpa, Tabasco localizado no México a cera dos meliponínios é utilizada para fins ritualísticos, através da preparação de velas e poções medicinais (Cano-Contreras et al. 2013).

\section{ConClusão}

A atividade de meliponicultura na região de Cícero Dantas-BA é praticada de forma tradicional, contando com técnicas simples de manejo e criação das abelhas em "cortiços", caixas rústicas e racionais, havendo preferência pelos cortiços. O principal produto de interesse dos meliponicultores é o mel, que é retirado dos enxames seguindo uma série de restrições, dentre elas a quantidade presente em cada colmeia, a época do ano, preferencialmente logo após a época de chuvas. Além disso, os meliponicultores levam em consideração o 
destino final do mel, bem como qual será sua utilização.

A venda é feita diretamente aos interessados, uma vez que a procura pelo produto é esporádica, além de que a disponibilidade do mel não é constante devido à produção irregular. O preço varia de espécie para espécie, isso pode ser justificado pela quantidade do mel produzido, bem como pelo valor cultural da espécie. Essa venda pode ser uma opção de renda para aumentar a fonte de renda dos meliponicultores.

O mel é o produto mais procurado, utilizado pela comunidade local principalmente no tratamento de gripes e enfermidades da garganta e boca. Registrou-se também o uso do pólen e da cera das abelhas nativas, com menor representatividade, uma vez que essa prática não é muito comum entre os meliponicultores e muitos desconhecem suas formas de utilização, fazendo uso apenas dos produtos que lhes foram apresentados ao longo da vida segundo os ensinamentos dos parentes ou pessoas próximas.

A atividade de meliponicultura apresenta valor cultural para a região, uma vez que a forma de criação e ao uso do mel, não seguem indicações técnicas em sua maioria, mas ocorrem seguindo o conhecimento prévio que foi adquirido através da prática cotidiana, bem como através de informações provenientes dos pais, avôs, parentes próximos ou pessoas da região.

\section{REFERÊNCIAS}

Albuqueruque PU, Lucena RFP e Alencar NL. 2010. Métodos e técnicas para coletas de dados etnobilógicos. In: Albuqueruque PU et at (Eds), Métodos e técnicas na pesquisa etnobiológica e etnoecológica, Recife, PE: NUPEAA, p. 41-64.

Alves RMO, Souza BA e Carvalho CAL. 2007. Notas sobre a bionomia de Melipona mandacaia (Apidae: Meliponina). Magistra, 19(3):204-212.

Amorozo MCM e Viertler RB. 2010. A abordagem qualitativa na coleta e análise de dados em etnobiologia e etnoecologia. In: Albuquerque UP et at (Eds). Métodos e técnicas na pesquisa etnobiológica e etnoecológica. Recife: NUPEEA, p. 65-83.

Badie MC. 2009. Uma etnografia sobre la Miel em la Cultura Mbya-Guaraní, Quito- Ecuador: Abya Yala, $147 \mathrm{p}$.

Bailey K. 1994. Methods of social research. 4nd ed., New York: The Free Press, 588 p.

Bardin L. 2011. Análise de conteúdo, São Paulo: Edições 70, 279 p.

Blásquez JR, Costa-Neto EM, Landero-Torres I. 2009. Comparación de especies de abejas comestibles en la Sierra de Jibóia, (Bahia, Brasil) y Sierra de Zongolica (Veracruz, México). Revista Colombiana de Entomología, 35(2):217-223.

Borsato DM, Esmerino LA, Farago PV, Miguel MD, Miguel OG. 2013. Atividade antimicrobiana de méis produzidos por meliponíneos nativos do Paraná (Brasil). Boletim Centro de Pesquisa de Processamento de Alimentos, 31(1):57-66. 
Camargo JMF, Posey DA. 1990. O conhecimento dos Kayapó sobre as abelhas sociais sem ferrão (Meliponidae, Apidae, Hymenoptera): notas adicionais. Boletim do Museu Paraense Emilio Goeldi, Série Zoologia, $6(1): 17-42$.

Cano-Contreras EJ, Martínez CM, AGuilar CCB. 2013. La "Abeja de Monte” (Insecta: Apidae, Meliponini) de los Choles de Tacotalpa, Tabasco: Conocimiento Local, Presente y Futuro. Etnobiología, 11(2):47-57.

Cortopassi-Laurino M, Macedo ERM. 1998. Vida da Abelha Jandaira Melipona subnitida. In: Anais do XII Congresso Brasileiro de Apicultura (Salvador, CBA), p. 65-67.

Cortopassi-Laurino M. 2005. A abelha jataí: uma espécie bandeira? (Tetragonisca angustula Latreille 1811). Revista Mensagem Doce, 80:34-38.

Cortopassi-Laurino M, Imperatriz-Fonseca VL, Roubik DW, Dollin A, Heard T, Aguilar IB, Venturieri GC, Eardley C, Nogueira-Neto P. 2006. Global Meliponiculture: challenges and opportunities. Apidologie, 37(2):275.

Carvalho RMA, Martins CF, Mourão JS. 2014. Meliponiculture in Quilombola communities of Ipiranga and Gurugi, Paraíba state, Brazil: an ethnoecological approach. Journal of Ethnobiology and Ethnomedicine, 10(3):1-12.

Costa-Neto EM. 1998. Folk Taxonomy and Cultural Significance of" Abeia"(Insecta, Hymenoptera) to the Pankarare, Northeastern Bahia State, Brazil. Journal of Ethnobiology, 18:1-13.

IBGE- CENSO. 2010. Instituto Brasileiro de Geografia e Estatística, Rio de Janeiro, 20(03):1-215.

Imperatriz-Fonseca VL, Contrera FAL, Kleinert AMP. 2004. A meliponicultura e a iniciativa brasileira dos polinizadores. In: XV Congresso Brasileiro Apicultura/I Congresso Brasileiro Meliponicultura (Natal$\mathrm{RN}): 1-7$.

Kerr WE, Carvalho GA, Nascimento VA. 1996. Abelha Uruçu: Biologia, Manejo e Conservação. Belo Horizonte: Acangaú, 144 p.

Kerr WE. 1997. A importância da meliponicultura para o país. Biotecnologia Ciência \& Desenvolvimento, $1(3): 42-44$.

Lopes M, Ferreira JB, Santos G. 2005. Abelhas sem-ferrão: a biodiversidade invisível. Agriculturas, 2(4):7-9.

Lopes ZS, Nogueira EMS. 2012. Caracterização da produção de mel de abelhas sem ferrão (Apidae: Meliponina) como subsídio ao desenvolvimento da meliponicultura em comunidades rurais de Cícero Dantas, Bahia. Jornada de Iniciação Científica da UNEB, Anais da XVI Jornada de Iniciação Científica, Salvador: EDUNEB, 600 p.

Martins VS, Schiavetti A, Souto FJB. 2011. Ethnoecological knowledge of the artisan fishermen of octopi (Octopus spp.) in the community of Coroa Vermelha (Santa Cruz Cabrália, Bahia). Anais da Academia Brasileira de Ciências, 83(20):513-522.

Mercês MD, Peralta ED, Uetanabaro APT, Lucchese AM. 2013. Antimicrobial activity of honey from five 
species of Brazilian stingless bees. Ciência Rural, 43(4):672-675.

Nates-Parra G, Rosso-Londoño JM. 2013. Diversidad de abejas sin aguijón (Hymenoptera:Meliponini) utilizadas en meliponicultura en Colombia. Acta biológica Colombiana, 18(3):415-426.

Prado DE. 2003. As Caatingas da América do Sul. In: LEAL RI et al. (Eds). Ecologia e conservação da Caatinga. Recife: Editora Universitária da UFPE, 823 p.

Posey DA. 1978. Ethnoentomological survey of Amerind groups in lowland Latin America. The Florida Entomologist, 61(6):225-229.

Posey DA. 1982. The importance of bees to Kayapó Indians of the Brazilian Amazon. The Florida Entomologist, 65(4):452-458.

Posey DA. 1983. Ethnomethodology as an emic guide to cultural systems: the case of the insects and the Kayapó indians of Amazonia. Revista brasileira de Zoologia, 1(3):135-144.

Rosso JM, Imperatriz-Fonseca VL, Cortopassi-Laurino M. 2001. Meliponicultura en Brasil I: Situacion em 2001 y Perspectivas. Memórias del II Seminário Mexicano sobre Abejas sin Aguijón, Mérida, México, p. 28-35.

Sampaio JA, Castro MS, Silva FO. 2009. Uso da cera de abelhas pelos índios Pankararé no Raso da Catarina, Bahia, Brasil. Arquivos do Museu Nacional, 67(1-2):3-12.

SEI-BA - Superintendência de Estudos Econômicos e Sociais da Bahia. 2011. Estatística dos municípios baianos. Secretaria de Planejamento e Ciência Tecnológica, n. 25, p. 73-90.

Souza RCS, Yuyama LKO, Aguiar JPL, Oliveira FPM. 2004. Valor nutricional do mel e pólen de abelhas sem ferrão da região amazônica. Acta Amazonica, 34(2):333-336.

Torres A, Garadew A, Schmolz E, Lamprecht I. 2004. Calorimetric investigation of the antimicrobial action and insight into the chemical properties of "angelita" honey-a product of the stingless bee Tetragonisca angustula from Colombia. Thermochimica Acta, 415(1):107-113.

Venturieri GC, Raiol VFO, Pereira CAB. 2003. Avaliação da introdução da criação racional de Melipona fasciculata (Apidae: Meliponina), entre os agricultores familiares de Bragança - PA, Brasil. Biota Neotropica, 3(2):1-7.

Venturieri GC. 2008. Criação racional de meliponíneos: uma alternativa econômica entre os agricultores familiares amazônicos. Mensagem Doce, 96.

Villanueva GR, Roubik DW, Colli-Ucán W. 2005. Extinction of Melipona beecheii and traditional beekeeping in the Yucatán Peninsula. Bee World, 86(2):35-41.

Yamamoto DY, Akatsu IP, Soares AEE. 2007. Quantificação da produção do mel de Scaptotrigona aff. depilis (Hymenoptera, Apidae, Apinae) do município de Luiz Antonio, São Paulo, Brasil. Bioscience Journal, 23(1):8993. 